\title{
Three-dimensional speckle tracking echocardiography for the evaluation of segmental myocardial deformation
}

\author{
Janine Baum, Florian Beeres, Silke Van Hall, Yang Chul Boering, Eva Susanne Kehmeier, Tobias \\ Zeus, Christian Meyer, Tienush Rassaf, Malte Kelm, Jan Balzer \\ Department of Cardiology, Pneumology and Angiology, University Hospital Duesseldorf, Duesseldorf, Germany \\ Correspondence: Janine Baum. Address: Department of Cardiology, Pneumology and Angiology, University Hospital \\ Duesseldorf, Moorenstrasse 5, D-40225 Duesseldorf, Germany. E-mail: Janine.Baum@med.uni-duesseldorf.de
}

Received: October 10, 2013

Accepted: January 15, 2014

Online Published: February 21, 2014

DOI : $10.5430 / j b g c . v 4 n 2 p 23$

URL: http://dx.doi.org/10.5430/jbgc.v4n2p23

\begin{abstract}
Background: Although the feasibility of three-dimensional (3D) speckle tracking echocardiography (STE) for the evaluation of myocardial function has been demonstrated, the poor reproducibility of strain measurements obtained with 3D STE as compared to two-dimensional (2D) STE has been controversially discussed. Aim of this study was to demonstrate the benefit of longitudinal strain analysis by 3D STE as compared to the established 2D STE techniques.
\end{abstract}

Methods: 2D and 3D STE was performed in 30 volunteers with normal systolic left ventricular (LV) function using cardiac ultrasound systems from two different vendors (Vivid E9 and iE33 xMATRIX). Global and segmental longitudinal strain (GLS, SLS) values were analyzed for 2D STE using respective software packages (Vivid E9: EchoPAC AFI; iE33 xMATRIX: QLAB CMQ 9.0). Measurements for 3D STE were performed using specific software for Vivid E9 (EchoPAC 4DAutoLVQ) and unspecific software for iE33 xMATRIX (TomTec Imaging Systems 4D left ventricular Analysis). Intra-, interobserver and test-retest variability as well as times for acquisition and analysis were compared between 2D and 3D STE techniques.

Results: The reliability of SLS measurements using 3D STE was non-inferior to the measurements obtained by 2D STE, with perpetual constant results in all tests (ICC SLS 3D $0.78-0.94$ vs. SLS 2D $0.73-0.93$ ). Agreements between SLS values were better when vendor specific 2D and 3D software was applied. GLS measurements showed inhomogeneous results for both techniques (ICC GLS 3D $0.40-0.93$ vs. GLS 2D $0.13-0.91$ ). Acquisition time was significantly shorter for 3D datasets than for 2D datasets (3D $51.0 \pm 10.66$ sec vs. 2D $91.0 \pm 10.9$ sec, $p<.01$ ).

Conclusion: 3D STE is a time-saving technology for the evaluation of myocardial deformation in daily clinical practice, generating results that are comparable to the conventional 2D STE techniques. SLS obtained by 3D STE seems to be a more robust parameter than GLS for the assessment of myocardial deformation, especially when vendor specific software packages are used.

\section{Key words}

Echocardiography, Left ventricular function, Three-dimensional echocardiography, Strain 


\section{Introduction}

The assessment of left ventricular (LV) volumes and function so far was restricted to direct volumetric analysis based on endocardial excursion, whereas assessment of actual myocardial deformation was only possible with techniques such as Tissue Doppler Imaging (TDI) and two-dimensional (2D) speckle-tracking echocardiography (STE). Although TDI has been shown feasible for the assessment of myocardial function and deformation, these measurements are limited by its angle dependency and therefore often are difficult to apply in clinical practice ${ }^{[1]}$. Strain analysis by 2D STE has emerged as a quantitative technique to solve this problem ${ }^{[2]}$, and has been validated against sonomicrometry and magnetic resonance imaging ${ }^{[3-5]}$. It could be demonstrated that 2D STE is a sufficient technique to detect even minimal changes in cardiac function that cannot be detected by traditional techniques ${ }^{[6-8]}$. Pathognomonic changes in deformation patterns measured by 2D STE are described ${ }^{[9]}$ and the technique today is irreplaceable for mechanical dyssynchrony assessment ${ }^{[10]}$. Reliability and inter-vendor changeability of 2D STE has been studied recently in large numbers of subjects showing moderate to good results for global longitudinal strain (GLS) analysis ${ }^{[11-13]}$. However, 2D STE is limited by its two-dimensional nature, such as the loss of speckles moving out the image plane and foreshortening of apical views and its time-consuming data-acquisition and analysis ${ }^{[14]}$. For that reason the technique is not widely accepted in clinical routine use. Three-dimensional (3D) STE has been shown to overcome these limitations, enabling the analysis of myocardial deformation in its longitudinal, circumferential and radial direction within a single 3D volume set ${ }^{[15-17]}$.

Although 3D STE is increasingly used for the evaluation of myocardial deformation, its implementation into clinical guidelines so far was hampered by its inhomogeneous results reported in recent studies ${ }^{[18,19]}$ and its lack of standardization among manufacturers ${ }^{[19]}$. The aim of this study was to demonstrate the benefit of longitudinal strain analysis by 3D STE as compared to 2D STE and its capability in daily clinical practice. To our knowledge we are the first to study both inter-vendor and inter-method agreement of 2D and 3D STE within one study-protocol.

\section{Patients and methods}

\subsection{Study population}

Thirty healthy volunteers with normal LV function as determined by conventional 2D echocardiography were prospectively studied (18 men, 12 women; mean age $27 \pm 10$ years). The protocol was approved by the institutional review board, and written informed consent was obtained in all subjects.

\subsection{Echocardiography}

Echocardiography was performed in the left lateral decubitus position using a Vivid E9 (GE-Healthcare, Horten, Norway; vendor 1, V1). We obtained brightness-mode (B-mode) images in apical four-, two- and three-chamber views and continuous wave (CW) Doppler profiles of aortic blood-flow. Image contrast, depth and sector size were optimized and care was taken not to foreshorten views in order to include the complete myocardial wall during the cardiac cycle and to have an optimal setting of 50-70 frames per second. Subsequently a minimum of two 3D datasets was obtained with a matrix-array transducer. Probands were asked to follow breathing commands to achieve a multi-beat 3D volume of a minimum of four heartbeats without artefacts. In the same session we obtained conventional 2D images and 3D datasets by a second ultrasound system, iE33 xMATRIX (Philips Healthcare, Andover, USA; vendor 2, V2). Images were stored digitally for further offline-analysis. All data were acquired by the same experienced echocardiographer. Figure 1 demonstrates the study-design with derived abbreviations.

\subsection{I mage analysis}

2D and 3D datasets of V1 were analysed by the vendor specific software (S1) (2D: AFI, EchoPAC v110.1.3, GE-Healthcare, Horten, Norway and 3D: 4DAutoLVQ, EchoPAC v110.1.3, GE-Healthcare, Horten, Norway). For 2D analysis manual time-settings were acquired at end-systole in the CW Doppler profile of aortic blood-flow. The best apical 
3-chamber view image was chosen to start the 2D strain analysis. After placement of three orientation points, endocardial contours were detected automatically, including the papillary muscle into the LV cavity. For the definition of the regions of interest, the LV wall was sub-divided into seven segments, including the LV apex. If necessary, endocardial and epicardial borders were adjusted manually. Following the myocardial motion frame by frame, left ventricular contraction was tracked automatically during the cardiac cycle. A visual control of the tracking was performed to ensure accurate motion of the displayed segments according to their belonging myocardial section. If the tracking was satisfactory, 2- and 4-chamber view images were analyzed following the same principle. Finally, measurements were displayed in a 17 segments bulls-eye, and global results were calculated automatically.

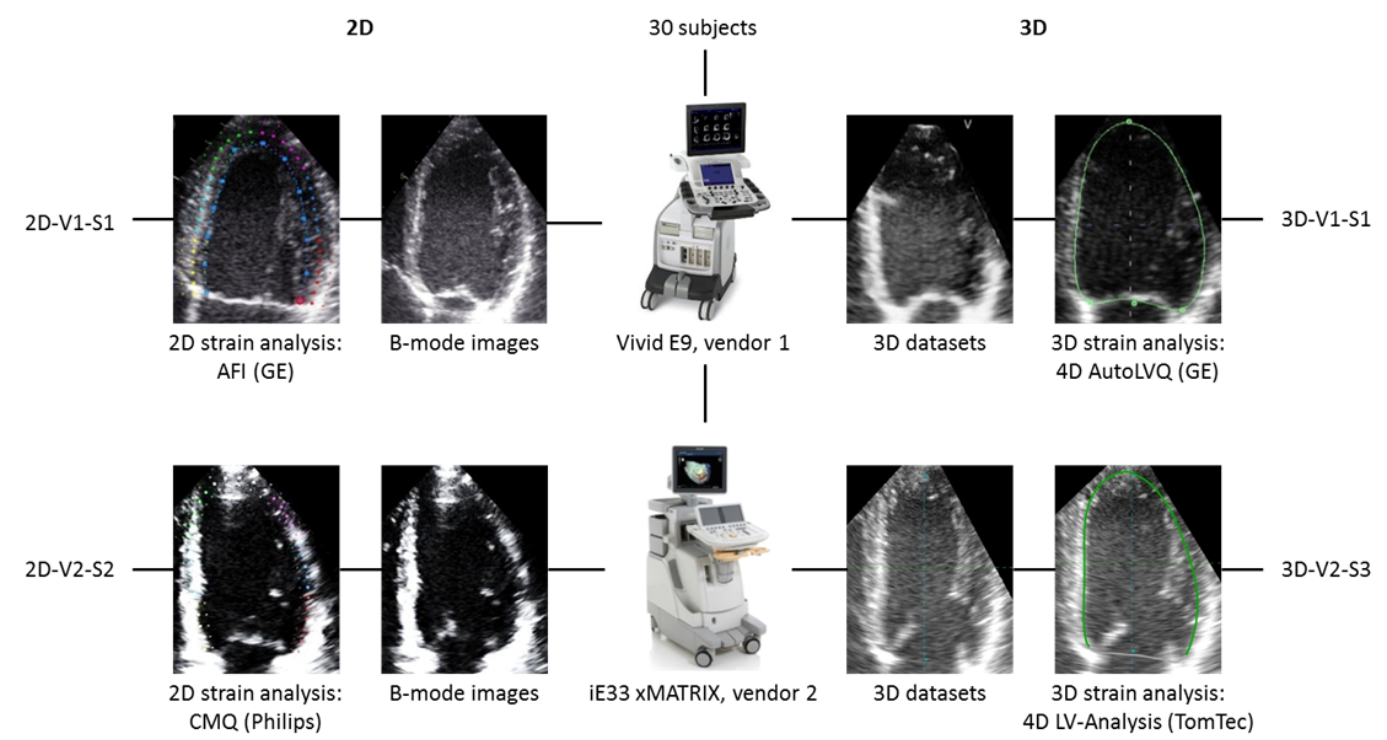

Figure 1. Flow-chart of the study design. 2D, 2-dimensional; 3D, 3-dimensional; V1, vendor 1: Vivid E9 GE-Healthcare, Horten, Norway; V2, vendor 2: iE33 xMATRIX Philips Healthcare, Andover, USA; S1, software 1: (2D) AFI, Automated function imaging, (3D) 4DAutoLVQ EchoPAC v110.1.3, GE-Healthcare, Horten, Norway; S2, software 2: (2D) QLAB CMQ 9.0, Philips Healthcare, Andover, USA; S3, software 3: (3D) 4D LV-Analysis, TomTec Imaging Systems, Unterschleißheim, Germany.

Figure 2. (a) 3D dataset displayed in apical 4-chamber view, 2-chamber view, 3-chamber view and short axis views for longitudinal strain analysis. After automatic boarder-detection and manual adaption, segment positions are demonstrated (narrow lines). Results of segmental 3D speckle tracking analysis are displayed as curve diagrams (b) and in terms of a 17-segment bulls-eye model (c). ED, end-diastole; ES, end-systole; G, Global longitudinal strain

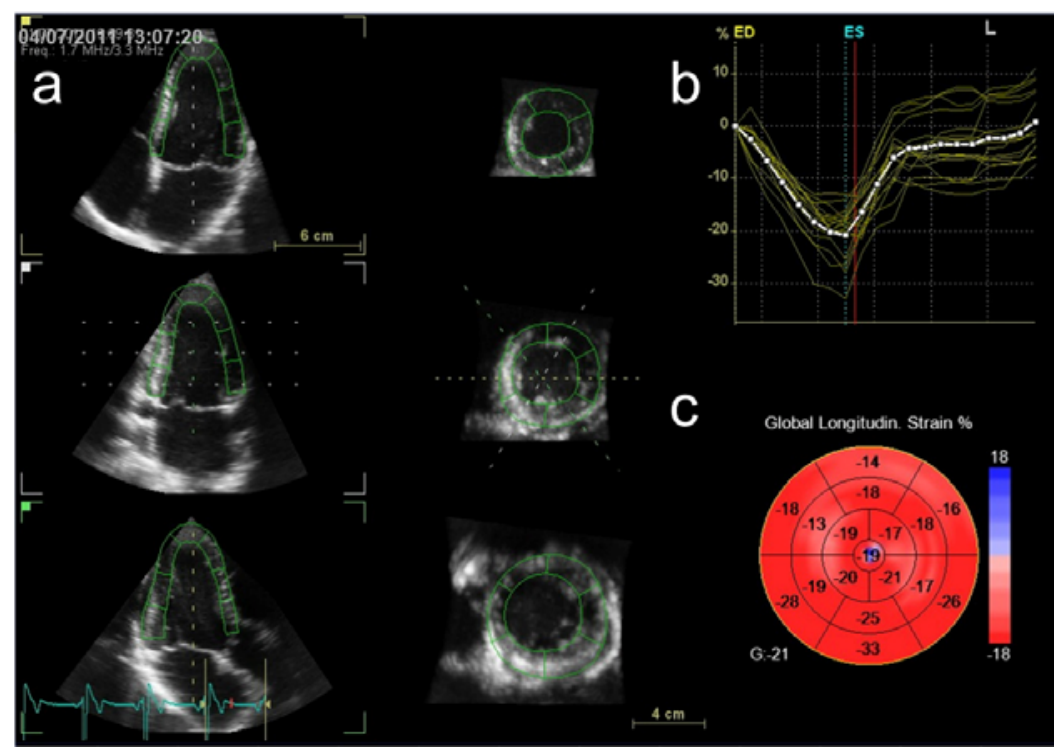

For 3D analysis, volume datasets were displayed as conventional apical and short axis views in a single screen (see Figure 2). Two orientation points were designated to the mitral valve level and the LV apex in end-diastole and Published by Sciedu Press 
end-systole. Endocardial contours were detected automatically and were adjusted if necessary. The motion of the 3D myocardium was tracked throughout the cardiac cycle and deformation parameters were calculated for each segment. Tracking was proofed visually and, if not accurate, repeated after manual adjustment. Completing the analysis, a 17 segments bulls-eye with global results was displayed.

2D images of V2 were also analyzed by the vendor specific software (S2) (QLAB CMQ 9.0, Philips Healthcare, Andover, USA), following the principles described above. In contrast, end-systolic time-settings were performed by visual control of aortic valve closure in the apical 3-chamber view. 3D datasets of V2 were analyzed by a vendor unspecific software (S3) (4D LV-Analysis, TomTec Imaging Systems, Unterschleißheim, Germany).

\subsection{I ntra- and inter-observer variability and test-retest reliability}

Intra- and interobserver variability were assessed for every vendor and software by repeated analysis of the included loops of 10 subset volunteers by the same observer and by another experienced observer blinded to previous results. Intraobserver analysis was performed at least one week apart from the first measurement.

To assess test-retest reliability, the complete echocardiographic and analysis protocol was performed again in the subgroup of 10 volunteers, after a minimum time-period of one month.

\subsection{Statistical analysis}

Statistical analysis was performed with standard software (MedCalc Software Version 10.3.0.0, Acacialaan 22, B-8400 Ostend, Belgium). Continuous data are presented as mean \pm standard deviation. All hypotheses were constructed as two tailed, and $p$-values $<.05$ were considered statistically significant. Reproducibility was evaluated by intra class correlation coefficients (ICC) for intra- and interobserver as well as test-retest analysis. Bland-Altman analysis was performed to assess inter-method agreement between 2D and 3D techniques as well as different 2D and 3D methods.

\section{Results}

Three of the 30 volunteers were excluded due to bad acoustic windows. Among the 27 included subjects, average image frame rate was $56.1 \pm 0$ frames/sec for 2D-V1-S1 analysis, $50 \pm 0$ frames/sec for 2D-V2-S2 analysis, $41.7 \pm 10.9$ volumes/sec for 3D-V1-S1 analysis and $43.1 \pm 8.9$ volumes/sec for 3D-V2-S3 analysis. Mean heart rate was $62.5 \pm 10.2$ beats/min. Mean values of GLS and segmental longitudinal strain (SLS) measurements are shown in Table 1.

Table 1. Mean global- and segmental longitudinal strain values

\begin{tabular}{lllll}
\hline & 2D-V1-S1 (\%) & 3D-V1-S1 (\%) & 2D-V2-S2 (\%) & 3D-V2-S3 (\%) \\
\hline Mean GLS $\pm S D$ & $-19.56 \pm 1.70$ & $-19.78 \pm 2.45$ & $-19.81 \pm 2.99$ & $-18.07 \pm 3.17$ \\
Mean SLS $\pm S D$ & $-19.60 \pm 4.34$ & $-19.95 \pm 5.43$ & $-20.97 \pm 6.59$ & $-18.17 \pm 5.00$ \\
\hline
\end{tabular}

Note. Mean values of global- and segmental longitudinal strain (GLS, SLS) analysis of 2-dimensional (2D) and 3-dimensional (3D) speckle tracking echocardiography measured by different vendors and software. SD, standard deviation; 2D-V1-S1, 2D-vendor 1-software 1; 3D-V1-S1, 3D-vendor 1-software 1; 2D-V2-S2, 2D-vendor 2-software 2; 3D-V2-S3, 3D-vendor 2-software 3

\subsection{Agreement between 2D and 3D STE techniques}

Figure 3 demonstrates the Blant-Altman plots for SLS measurements. Figure 3a illustrates deviating results comparing 2D and 3D SLS measurements from two different vendors. Similar results are shown for the inter-method comparison between 2D and 3D techniques by different vendors (see Figure 3b). A good agreement for SLS analysis could only be demonstrated for 2D and 3D measurements by the same vendor (V1) and its related software, with low bias and narrow limits of agreement (bias 0.3 , limits of agreement \pm 11.2 ). The comparison of $2 \mathrm{D}$ and $3 \mathrm{D}$ measurements by $\mathrm{V} 2$ also showed divergent results (see Figure 3c). 
Figure 3. Agreement between segmental longitudinal strain measurements by 2-dimensional (2D) and 3-dimensional (3D) software of two different vendors (a, inter-vendor), by 2D software and 3D software of different vendors (b, inter-method and inter-vendor) and by 2D software and $3 \mathrm{D}$ software of the same vendor (c, inter-method).

2D-V1-S1, 2D-vendor 1-software 1; 3D-V1-S1, 3D-vendor 1-software 1; 2D-V2-S2, 2D-vendor 2-software 2; 3D-V2-S3, 3D-vendor 2-software 3

Figure 4. Comparison of global longitudinal strain measured by 2dimensional (2D) and 3-dimensional (3D) software of two different vendors (a, inter-vendor), by 2D software and 3D software of different vendors (b, inter-method and inter-vendor) and by $2 \mathrm{D}$ software and 3D software of the same vendor (c, inter-method). 2D-V1-S1, 2D-vendor 1-software 1; 3D-V1-S1, 3D-vendor 1-software 1; 2D-V2-S2, 2D-vendor 2-software 2; 3D-V2-S3, 3D-vendor 2-software 3
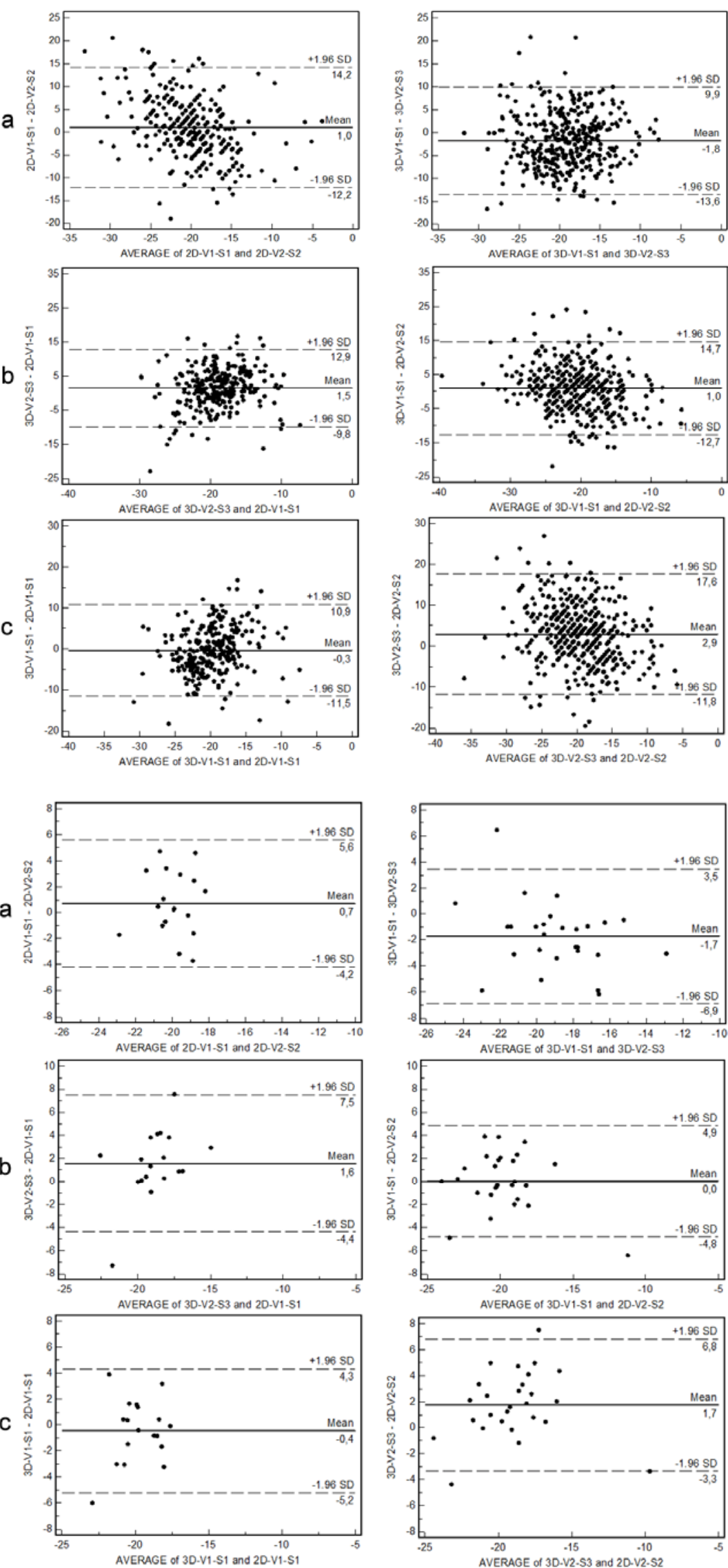
Regarding the Blant-Altman plots of GLS analysis, we could demonstrate a satisfactory agreement of inter-method and inter-vendor comparison with low bias and narrow limits of agreement (bias $0.0-1.7$, limits of agreement \pm 4.9 to \pm 6.0 , see Figure 4a-c).

\subsection{Reliability of 2D and 3D STE for longitudinal strain analysis}

The results of the intra- and interobserver variability and the test-retest reliability are displayed in Table 2. For GLS analysis we could identify a good correlation for V1 and a weak correlation for V2 (averaged ICC of all tests: 2D-V1-S1 0.73 vs. 2D-V2-S2 0.62). GLS measurements by 3D STE appear to be very inhomogeneous (ICC 3D-V1-S1 $0.48-0.93$, 3D-V2-S3 $0.47-0.92$ ). We could demonstrate the best reliability for SLS measurements with constant good results in all tests using 3D SLS analysis by V1, followed by 2D SLS analysis by V1 (averaged ICC of all tests: 3D-V1-S1 0.84 and 2D-V1-S1 0.82).

Table 2. Intra- and interobserver variability and test-retest reliability

\begin{tabular}{lllll}
\hline & 2D-V1-S1 (ICC) & 3D-V1-S1 (ICC) & 2D-V2-S2 (ICC) & 3D-V2-S3 (ICC) \\
\hline Global longitudinal Strain & & & & \\
Intraobserver & 0.91 & 0.93 & 0.85 & 0.92 \\
Interobserver & 0.70 & 0.40 & 0.88 & 0.91 \\
Test-retest & 0.57 & 0.48 & 0.13 & 0.47 \\
Segmental longitudinal Strain & & & & \\
Intraobserver & 0.93 & 0.94 & 0.71 & 0.79 \\
Interobserver & 0.81 & 0.81 & 0.69 & 0.71 \\
Test-retest & 0.73 & 0.78 & 0.50 & 0.64 \\
\hline
\end{tabular}

Note. Reliability of global and segmental strain measurements by 2-dimensional (2D) and 3-dimensional (3D) speckle tracking echocardiography presented as intra class correlation coefficient (ICC) of intra- and interobserver variability and test-retest analysis. 2D-V1-S1, 2D-vendor 1-software 1; 3D-V1-S1, 3D-vendor 1-software 1; 2D-V2-S2, 2D-vendor 2-software 2; 3D-V2-S3, 3D-vendor 2-software 3

\subsection{Applicability of 3-dimensional speckle tracking echocardiography}

Acquisition time of 3D volume datasets was significantly shorter as compared to conventional 2D apical 4-, 3- and 2-chamber views (3D-V1-S1 and 3D-V2-S3 $51.0 \pm 10.66$ sec vs. 2D-V1-S1 and 2D-V2-S2 $91.0 \pm 10.9$ sec, $p<.01$, see Figure 5). Analysis time did not differ significantly between 2D and 3D strain-analysis (3D-V1-S1 and 3D-V2-S3 $3.89 \pm$ $1.1 \mathrm{~min} v$ s. 2D-V1-S1 and 2D-V2-S2 $4.06 \pm 1.8 \mathrm{~min}, p=.76$, see Figure 6).

Figure 5. Acquisition time of 2-dimensional (2D) and 3-dimensional (3D) datasets. Statistical significance vs. 2D-V1-S1 and 2D-V2-S2: * $p<.01$. Sec, seconds; 2D-V1-S1, 2D-vendor 1-software 1; 3D-V1-S1, 3D-vendor 1-software 1; 2D-V2-S2, 2D-vendor 2-software 2; 3D-V2-S3, 3D-vendor 2-software 3

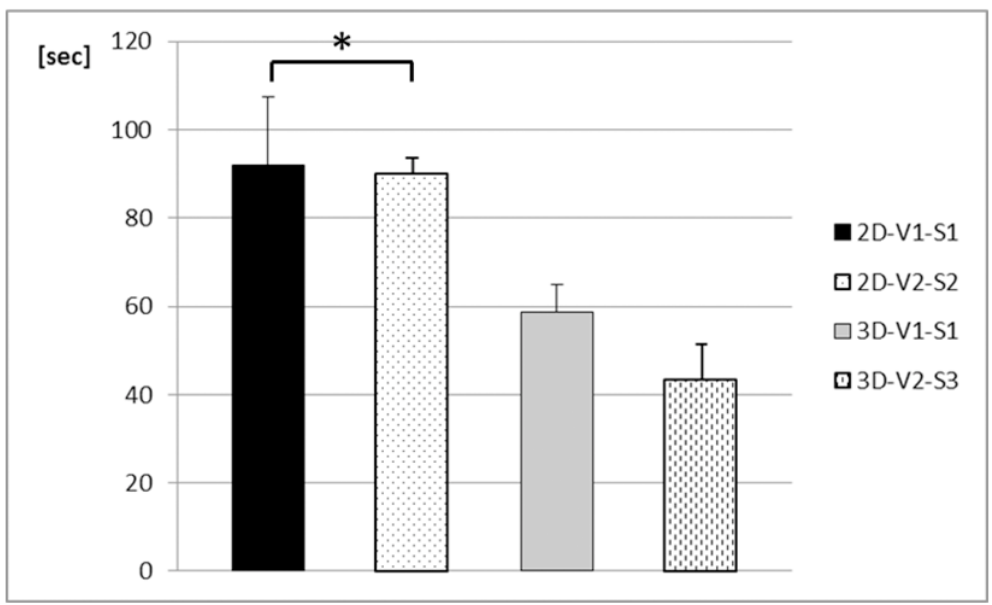


Figure 6. Time of 2-dimensional (2D) and 3-dimensional (3D) strain-analysis. Min, minutes; 2D-V1-S1, 2D-vendor 1-software 1; 3D-V1-S1, 3D-vendor 1-software 1; 2D-V2-S2, 2D-vendor 2-software 2; 3D-V2-S3, 3D-vendor 2-software 3

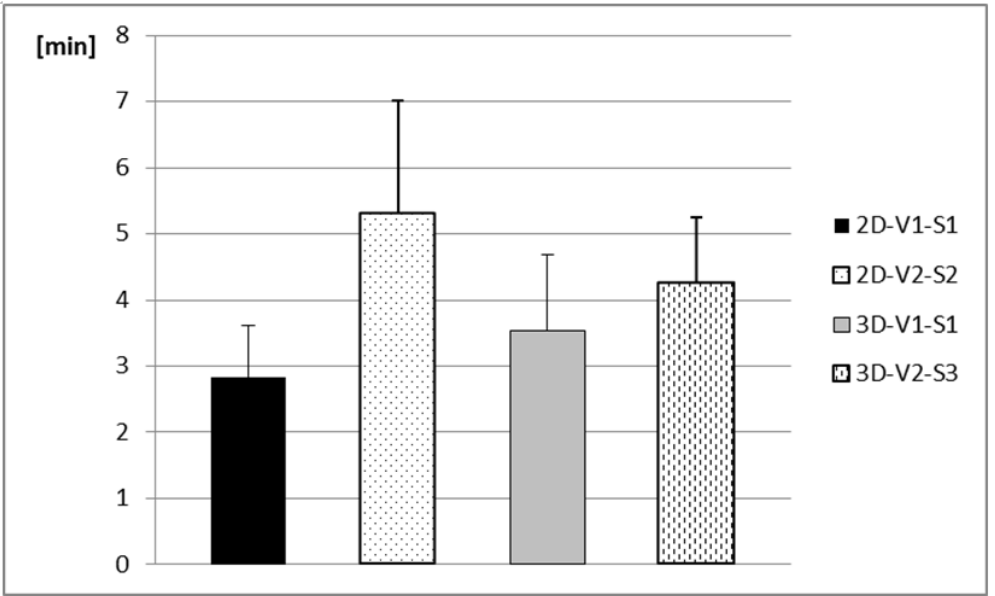

\section{Discussion}

The major findings of the present study are:

- The evaluation of myocardial deformation by 3D STE is a feasible technique and generates results that are comparable to 2D STE, with the prerequisite of using the same vendor and its specific software.

- SLS obtained by 3D STE seems to be a more robust parameter than GLS for the assessment of myocardial deformation, especially when vendor specific software packages are used.

- 3D STE is a time-saving technology for the evaluation of myocardial deformation in daily clinical practice.

\subsection{Agreement between 2D and 3D STE techniques}

A substantial problem in deformation imaging is the lack of interchangeability between different vendors ${ }^{[20]}$. Our findings demonstrate comparable values for GLS measurements by different techniques (2D and 3D STE) as well as by different vendors, as reported in previous studies ${ }^{[11-13,21-23]}$. Inter-method comparison of SLS measurements is only reported in a few studies. Gayat et al. published a study in 2011, evaluating the inter-vendor variability for 3D STE. The authors could demonstrate that the inter-vendor comparison of SLS values showed systematically two- or three-times wider limits of agreement as compared to GLS measurements, while biases were similar or worse ${ }^{[19]}$. Similar results could be identified in our study with wide limits of agreement comparing SLS measurements from 3D STE and additionally 2D STE by two different vendors. Gayat et al. used a software package that enables an analysis of 3D datasets of two different vendors. They could demonstrate a better inter-technique agreement by using the same software, compared to an analysis of datasets from the same vendor with a different software. We discovered best agreements of SLS analysis using 2D and 3D analysis protocols from the same vendor. Worst results could be demonstrated comparing inter-method (2D and 3D) and inter-vendor (Vivid E9 and iE33 xMATRIX) measurements. Our findings confirm the conclusion of Gayat et al. that analysis software has a stronger influence on measured values than vender specific datasets. Strain measurement by 2D STE is limited in its accuracy due to the impossibility to track the third dimension of the complex 3D motion of the myocardium. Even in a consecutive analysis of all conventional apical B-mode images, results will only represent sections of the spatial organ ${ }^{[24]}$. Several studies report longitudinal strain measurements to be more robust as compared with radial and circumferential parameters ${ }^{[23]}$. In longitudinal strain analysis of B-mode images, the direction of myocardial contraction follows mainly a straight line from ventricular base to the apex. Therefore longitudinal strain is least influenced by out of plane motion of speckles ${ }^{[23]}$. A study of Maffessanti et al. reported worse results comparing longitudinal strain measurements by 2D and 3D STE in a mixed collective of 32 healthy volunteers and patients with cardiac disease ${ }^{[25]}$. In contrast Pérez de Isla et al. could demonstrate a high correlation for GLS analysis and modest 
correlation for SLS analysis between 2D and 3D STE of the same vendor in a similar cohort ${ }^{[24]}$. The Blant-Altman analysis of our study constitutes better agreements of both 3D and 2D strain analysis for GLS and for SLS as compared with previous studies. Maffessanti et al. used 3D datasets with a temporal resolution of 11 to 23 volumes per second. With $40.1 \pm 11.5$ volumes/sec in our study 3D datasets had a much higher rate resulting in a better tracking and therefore more accurate results.

\subsection{Reliability of 2D and 3D STE for longitudinal strain analysis}

Within the last years the reliability of LV stain analysis by 2D STE was a focal point in numerous studies, presenting a good reproducibility of GLS and SLS measurements ${ }^{[13,22,26-29]}$. Using two different vendors in our study, we represent a good reliability of GLS and SLS measurements by 2D STE for V1. On the other hand, we could demonstrate only a weak reliability for 2D GLS and SLS measurements by V2. For strain analysis by 2D-V2-S2, we determined the longest analysis-time with $5.3 \pm 1.7 \mathrm{~min}$. The reason for the extended time-period was the necessity for multiple manual corrections, especially in defining the correct region of interest. Whenever manual adaption is required, the benefit of automatic border detection disappears and therewith strain measurement becomes more influenced by the individual observer, precluding a good reproducibility of the applied method. The high reliability of 3D STE for LV volume and ejection fraction measurements has already been demonstrated in a large number of subjects ${ }^{[18,30,31]}$. So far only few studies have evaluated the reliability of 3D STE for global and segmental LV strain analysis. Badano et al., Kleijn et al. and Gayat et al. could demonstrate a good intra- and interobserver agreement for GLS measurements by 3D STE ${ }^{[15,18,19]}$ using different vendors and software. We could identify a good intra- and interobserver variability for GLS measurements by V2, while there was only a weak correlation in interobserver and test-retest reliability of V1. For segmental measurements by 3D STE Kleijn et al. and Gayat et al. reported good intraobserver agreement, while interobserver agreement was shown to be less accurate. Our study demonstrates 3D STE to be equivalent for regional longitudinal strain analysis as compared to the reliability of 2D STE, with constant good results in intra- and interobserver variability and test-retest reliability using V1. Furthermore, GLS is calculated as an average of 17 segmental values. While averaged values differ for the reason of regional mistracking, for example by deviant manual endocardial border adaption, the segment-to-segment-correlation shows a better reliability. In this context, we could find a superior correlation of intra- and interobserver measurements comparing every single segment, emerging the accuracy of SLS analysis by 3D STE using V1. On the one hand, vendors differ in the storage format of their 3D datasets and the analysis software may be programmed in different algorithms (e.g. for myocardial border detection) and may also vary in defining the measured parameters ${ }^{[19]}$. This leads to disparate analysis-quality and therefore inhomogeneous findings concerning the reliability of 3D and 2D STE techniques.

\subsection{Applicability of 3-dimensional speckle tracking echocardiography}

The fast generation of a pyramidal 3D volume set can replace the elaborate acquisition of multiple B-mode images, as demonstrated in significant shorter acquisition times for 3D datasets by both vendors. Time of analysis for 3D STE did not differ significantly to 2D strain-analysis within the same vendor. However, following the 3D analysis protocol, important information such as LV volume, mass and ejection fraction, is additionally calculated in a single analysis cycle. Recent studies demonstrate the superiority of volume measurements by 3D STE as compared to 2D STE using magnetic resonance imaging as the reference technique ${ }^{[30-32]}$. Our study underlines previous findings of 3D STE being a faster and more complete tool for LV function-analysis ${ }^{[24,25]}$.

\subsection{Limitations}

With a mean age of $27 \pm 10$ years we studied a young and healthy collective resulting in better image quality than in many clinical routine patients. Another study limitation is the lack of a study population with cardiac disease resulting in global or regional LV dysfunction. Furthermore, our results were not compared with an independent reference technique, such as magnetic resonance imaging. It is therefore impossible to prove the accuracy of any of the four applied techniques. We therefore have to refer to previous studies, in which 2D STE was validated against sonomicrometry or magnetic resonance imaging. 


\section{Conclusion}

Our results demonstrate that 3D STE is a time-saving technology for the evaluation of myocardial deformation in daily clinical practice, generating results that are comparable to the conventional 2D STE techniques using the same vendor and its specific software. In this context, the accuracy of segmental longitudinal strain values obtained by 3D STE seems to be higher than for global longitudinal strain measurements, though demonstrating inhomogeneous results for both techniques. 3D STE is better than its reputation with the prerequisite that hard- and software for 2D and 3D analysis respectively have to be applied form the same manufacturer. Our paper underlines the need for multi-center studies investigating the diagnostic accuracy and reproducibility in various cardiac diseases on the one hand and the need for standardization of techniques, hard- and software for STE on the other hand. The results of our study disclose the future perspective of a complete LV function analysis by fully-automated software solutions, without the necessity of manual corrections.

\section{Conflict of interests}

The authors declare that they have no conflict of interests.

\section{Funding}

This study was supported with a restricted grant from the federal state government of North Rhine-Westphalia and the European Union (EFRE-Program “Med in.NRW”, support code 005-GW01-235A).

\section{References}

[1] Yu CM, Sanderson JE, Marwick TH. Tissue Doppler imaging a new prognosticator for cardiovascular diseases. J Am Coll Cardiol. 2007; 49: 1903-1914. PMid: 17498573. http://dx.doi.org/10.1016/j.jacc.2007.01.078

[2] Marwick TH. Measurement of strain and strain rate by echocardiography: ready for prime time? J Am Coll Cardiol. 2006; 47: 1313-1327. PMid: 16580516. http://dx.doi.org/10.1016/j.jacc.2005.11.063

[3] Seo Y, Ishizu T, Enomoto Y. Endocardial surface area tracking for assessment of regional LV wall deformation with 3D speckle tracking imaging. JACC Cardiovasc Imaging. 2011; 4: 358-365. PMid: 21492810. http://dx.doi.org/10.1016/j.jcmg.2010.12.007

[4] Amundsen BH, Helle-Valle T, Edvardsen T. Noninvasive myocardial strain measurement by speckle tracking echocardiography: validation against sonomicrometry and tagged magnetic resonance imaging. J Am Coll Cardiol. 2006; 47: 789-793. PMid: 16487846. http://dx.doi.org/10.1016/j.jacc.2005.10.040

[5] Cho GY, Chan J, Leano R. Comparison of two-dimensional speckle and tissue velocity based strain and validation with harmonic phase magnetic resonance imaging. Am J Cardiol. 2006; 97: 1661-1666. PMid: 16728234. http://dx.doi.org/10.1016/j.amjcard.2005.12.063

[6] Spethmann S, Dreger H, Schattke S. Two-dimensional speckle tracking of the left ventricle in patients with systemic sclerosis for an early detection of myocardial involvement. Eur Heart J Cardiovasc Imaging. 2012; 13: 863-870. PMid: 22427402. http://dx.doi.org/10.1093/ehjci/jes047

[7] Yip GW, Zhang Q, Xie JM. Resting global and regional left ventricular contractility in patients with heart failure and normal ejection fraction: insights from speckle-tracking echocardiography. Heart. 2011; 97: 287-294. PMid: 21193686. http://dx.doi.org/10.1136/hrt.2010.205815

[8] Shahul S, Rhee J, Hacker MR. Subclinical left ventricular dysfunction in preeclamptic women with preserved left ventricular ejection fraction: a 2D speckle-tracking imaging study. Circ Cardiovasc Imaging. 2012; 5: 734-739. PMid: 22891044. http://dx.doi.org/10.1161/CIRCIMAGING.112.973818

[9] Voigt JU, Exner B, Schmiedehausen K. Strain-rate imaging during dobutamine stress echocardiography provides objective evidence of inducible ischemia. Circulation. 2003; 107: 2120-2126. PMid: 12682001. http://dx.doi.org/10.1161/01.CIR.0000065249.69988.AA

[10] Voigt JU. Making a black box transparent. Eur Heart J Cardiovasc Imaging. 2013; 14: 201-202. PMid: 23087156. http://dx.doi.org/10.1093/ehjci/jes213

[11] Costa SP, Beaver TA, Rollor JL. Quantification of the variability associated with repeat measurements of left ventricular two-dimensional global longitudinal strain in a real-world setting. J Am Soc Echocardiogr. 2014; 27: 50-54. PMid: 24120317. http://dx.doi.org/10.1016/j.echo.2013.08.021

[12] Nelson MR, Hurst RT, Raslan SF. Echocardiographic measures of myocardial deformation by speckle-tracking technologies: the need for standardization? J Am Soc Echocardiogr. 2012; 25: 1189-1194. PMid: 22981227. http://dx.doi.org/10.1016/j.echo.2012.08.006 
[13] Risum N, Ali S, Olsen NT. Variability of global left ventricular deformation analysis using vendor dependent and independent two-dimensional speckle-tracking software in adults. J Am Soc Echocardiogr. 2012; 25: 1195-1203. PMid: 22981228. http://dx.doi.org/10.1016/j.echo.2012.08.007

[14] Mor-Avi V, Lang RM, Badano LP. Current and evolving echocardiographic techniques for the quantitative evaluation of cardiac mechanics: ASE/EAE consensus statement on methodology and indications endorsed by the Japanese Society of Echocardiography. Eur J Echocardiogr. 2011; 12: 167-205. PMid: 21385887. http://dx.doi.org/10.1093/ejechocard/jer021

[15] Badano LP, Cucchini U, Muraru D. Use of three-dimensional speckle tracking to assess left ventricular myocardial mechanics: inter-vendor consistency and reproducibility of strain measurements. Eur Heart J Cardiovasc Imaging. 2013; 14: 285-293. PMid: 22968525. http://dx.doi.org/10.1093/ehjci/jes184

[16] Mor-Avi V, Lang RM. Three-dimensional echocardiographic evaluation of the heart chambers: size, function, and mass. Cardiol Clin. 2007; 25: 241-251. PMid: 17765103. http://dx.doi.org/10.1016/j.ccl.2007.05.003

[17] Saito K, Okura H, Watanabe N. Comprehensive evaluation of left ventricular strain using speckle tracking echocardiography in normal adults: comparison of three-dimensional and two-dimensional approaches. J Am Soc Echocardiogr. 2009; 22: 1025-1030. PMid: 19556106. http://dx.doi.org/10.1016/j.echo.2009.05.021

[18] Kleijn SA, Aly MF, Terwee CB. Reliability of left ventricular volumes and function measurements using three-dimensional speckle tracking echocardiography. Eur Heart J Cardiovasc Imaging. 2012; 13: 159-168. PMid: 21926118. http://dx.doi.org/10.1093/ejechocard/jer174

[19] Gayat E, Ahmad H, Weinert L. Reproducibility and inter-vendor variability of left ventricular deformation measurements by three-dimensional speckle-tracking echocardiography. J Am Soc Echocardiogr. 2011; 24: 878-885. PMid: 21645991. http://dx.doi.org/10.1016/j.echo.2011.04.016

[20] Marwick TH. Consistency of myocardial deformation imaging between vendors. Eur J Echocardiogr. 2010; 11: 414-416. PMid: 20164088. http://dx.doi.org/10.1093/ejechocard/jeq006

[21] Altman M, Bergerot C, Aussoleil A. Assessment of left ventricular systolic function by deformation imaging derived from speckle tracking: a comparison between 2D and 3D echo modalities. Eur Heart J Cardiovasc Imaging 2013.

[22] Sun JP, Lee AP, Wu C. Quantification of left ventricular regional myocardial function using two-dimensional speckle tracking echocardiography in healthy volunteers--a multi-center study. Int J Cardiol. 2013; 167: 495-501. PMid: 22365315. http://dx.doi.org/10.1016/j.ijcard.2012.01.071

[23] Manovel A, Dawson D, Smith B. Assessment of left ventricular function by different speckle-tracking software. Eur J Echocardiogr. 2010; 11: 417-421. PMid: 20190272. http://dx.doi.org/10.1093/ejechocard/jep226

[24] Perez de Isla L, Balcones DV, Fernandez-Golfin C. Three-dimensional-wall motion tracking: a new and faster tool for myocardial strain assessment: comparison with two-dimensional-wall motion tracking. J Am Soc Echocardiogr. 2009; 22: 325-330. PMid: 19345302. http://dx.doi.org/10.1016/j.echo.2009.01.001

[25] Maffessanti F, Nesser HJ, Weinert L. Quantitative evaluation of regional left ventricular function using three-dimensional speckle tracking echocardiography in patients with and without heart disease. Am J Cardiol. 2009; 104: 1755-1762. PMid: 19962489. http://dx.doi.org/10.1016/j.amjcard.2009.07.060

[26] Oxborough D, George K, Birch KM. Intraobserver reliability of two-dimensional ultrasound derived strain imaging in the assessment of the left ventricle, right ventricle, and left atrium of healthy human hearts. Echocardiography. 2012; 29: 793-802. PMid: 22506912. http://dx.doi.org/10.1111/j.1540-8175.2012.01698.x

[27] Serri K, Reant P, Lafitte M. Global and regional myocardial function quantification by two-dimensional strain: application in hypertrophic cardiomyopathy. J Am Coll Cardiol. 2006; 47: 1175-1181. PMid: 16545649. http://dx.doi.org/10.1016/j.jacc.2005.10.061

[28] Mondillo S, Galderisi M, Mele D. Speckle-tracking echocardiography: a new technique for assessing myocardial function. J Ultrasound Med. 2011; 30: 71-83. PMid: 21193707.

[29] Marwick TH, Leano RL, Brown J. Myocardial strain measurement with 2-dimensional speckle-tracking echocardiography: definition of normal range. JACC Cardiovasc Imaging. 2009; 2: 80-84. PMid: 19356538. http://dx.doi.org/10.1016/j.jcmg.2007.12.007

[30] Nesser HJ, Mor-Avi V, Gorissen W. Quantification of left ventricular volumes using three-dimensional echocardiographic speckle tracking: comparison with MRI. Eur Heart J. 2009; 30: 1565-1573. PMid: 19482868. http://dx.doi.org/10.1093/eurheartj/ehp187

[31] Muraru D, Badano LP, Piccoli G. Validation of a novel automated border-detection algorithm for rapid and accurate quantitation of left ventricular volumes based on three-dimensional echocardiography. Eur J Echocardiogr. 2010; 11: 359-368. PMid: 20042421. http://dx.doi.org/10.1093/ejechocard/jep217

[32] Dorosz JL, Lezotte DC, Weitzenkamp DA. Performance of 3-dimensional echocardiography in measuring left ventricular volumes and ejection fraction: a systematic review and meta-analysis. J Am Coll Cardiol. 2012; 59: 1799-1808. PMid: 22575319. http://dx.doi.org/10.1016/j.jacc.2012.01.037 